\title{
THE STRUCTURAL BEHAVIOR OF SERRATED CAST-IN ANCHOR CHANNELS - A NUMERICAL STUDY ON THE LONGITUDINAL LOADING CLOSE TO THE EDGE
}

\author{
GEORG KARL KOCUR ${ }^{*}$, ELENI CHATZI ${ }^{\dagger}$ AND FRANK HÄUSLER ${ }^{*}$ \\ ${ }^{*}$ Halfen $\mathrm{GmbH}$ \\ Research-Development-Engineering \\ Langenfeld, Germany \\ e-mail: georg.kocur@halfen.de \\ e-mail: frank.haeusler@halfen.de \\ ${ }^{\dagger}$ ETH Zurich \\ Institute of Structural Engineering \\ Zurich, Switzerland \\ e-mail: chatzi@ibk.baug.ethz.ch
}

Key words: Finite Element Method, Computational Modeling, Concrete Fracture, Structural Concrete, Anchor Channels

\begin{abstract}
The structural behavior of concrete members due to cast-in anchor channels subjected to shear loading in longitudinal channel axis poses a non-trivial modeling task. The explicit applicability of recent calculation methods on anchor channels has rarely been validated by physical experiments. Preliminary test series on serrated anchor channels were carried out to determine the structural behavior under longitudinal shear loading. Anchor channels were installed in concrete close to the specimen's edge and positioned orthogonal to the edge. Each anchor channel was equipped with two riveted anchors. Loading was applied over serrated bolts until failure. Special attention was paid to the crack formation during loading. Of particular interest was the point in time when the load was redistributed from the front to the rear anchor. To attain a deeper insight with respect to the failure mechanisms, finite element simulations were performed. The main challenge, additionally to the complex mesh geometry, was in modeling the physical behavior of structural steel and concrete as a composite structure. The interaction between the brittle concrete and the ductile structural steel needs to be appropriately accounted for by means of adequate numerical simulation. Numerical calibration experiments, involving the structural steel members, were performed until satisfactory convergence was achieved. Load-deflection curves obtained from physical experiments were adopted for calibration of the numerical model. The obtained hybrid numerical/experimental results appear to contradict the results calculated by means of currently available design guidelines.
\end{abstract}




\section{INTRODUCTION}
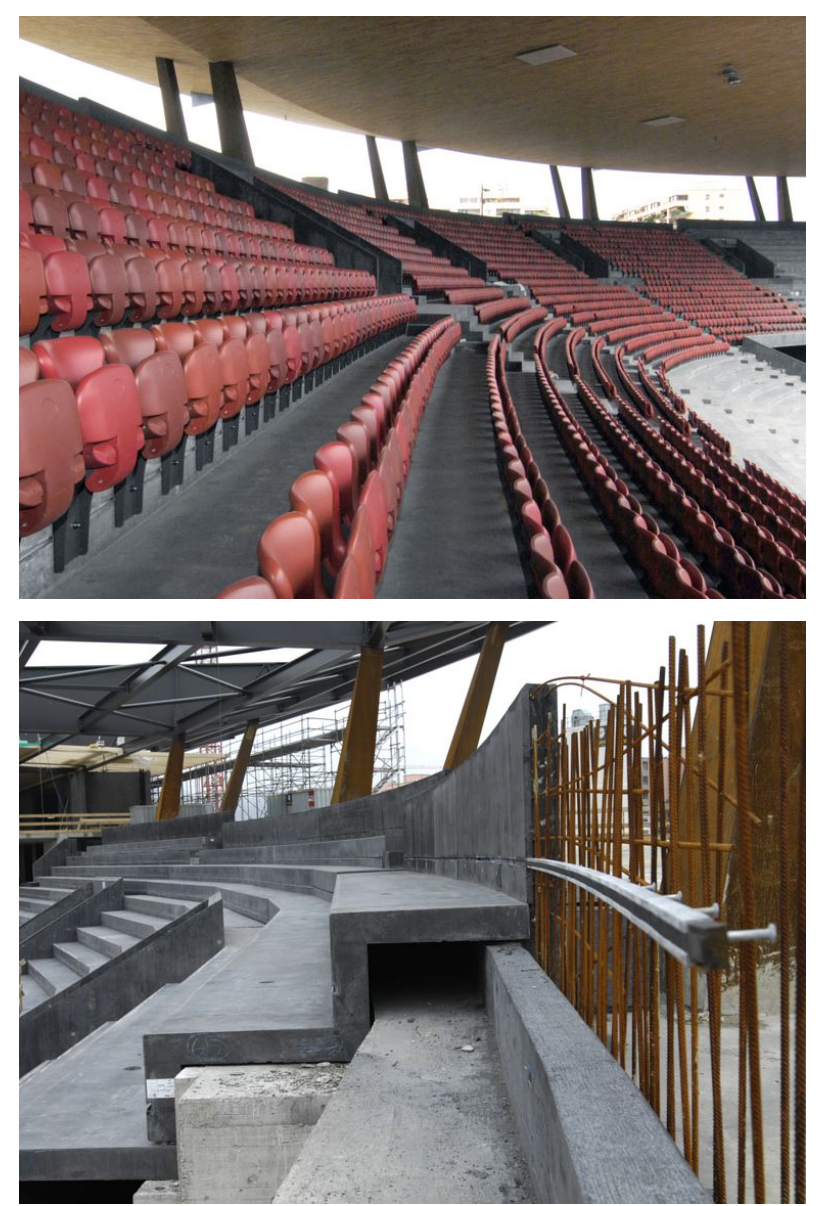

Figure 1: Application of anchor channels at grandstand of the Letzigrund Stadion in Zurich, Switzerland ${ }^{(C)}$ HALFEN.

Anchor channels provide a reliable, durable and adjustable connection to concrete. Typically these are cast-in concrete to avoid the need for post-installed anchors and field yielding. This minimizes the potential to damage the concrete or reinforcement during drilling. As observed in Figure 1 the anchor channels provide an elegant structural and architectural solution without distracting attention from the overall structure. Common areas of application include connections of curtain wall façades, elevators, masonry, civil engineering, and bridge installations.

The presented anchor channel type consists of a C-shaped steel channel profile, with serrated lips produced via hot-rolling and two round headed steel anchors (see Figure 2).
The round anchors are riveted to the channel back. The maximum number of anchors is not limited to a certain number, however in this study cases with two and three anchors are analyzed. Anchor channels can be easily mounted to timber or steel formwork through pre-punched holes in the back of the channel, not shown in detail here. The fixed channels remain flush with the surface and are not displaced during concrete pour. After hardening and when the concrete has reached the required compressive strength, the serrated hammerhead channel bolts (T-bolts), the washer and the hexagonal nut can be installed to fix the attachment (see Figure 3). To ensure optimal force transmission, installation torque is applied to the bolts. Embedded in concrete, the anchor channel can be loaded in tension (z-direction) and in shear, perpendicular ( $y$-direction), and longitudinal ( $x$-direction) to the channel axis. The loading type may be both static or dynamic (seismic and fatigue).

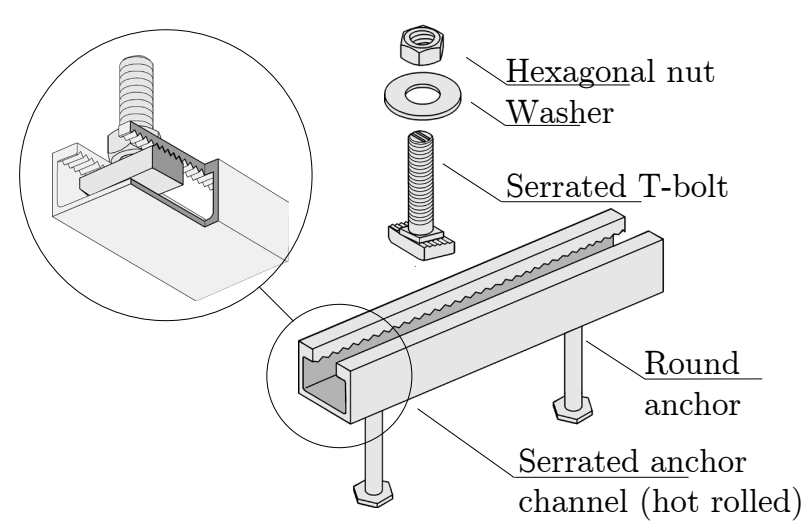

Figure 2: Anchor channel system

\section{PROBLEM STATEMENT}

The structural behavior of cast-in anchor channels located close to the edge and loaded in shear in the longitudinal channel axis poses a challenging simulation task. In addition, recent design guidelines [1-3] rely on approaches originally aimed for calculation of single and grouped headed fasteners connected by anchor plates. The explicit applicability of the calculation methods on anchor channels has rarely been validated by physical experiments. 
Lack of clarity motivated the presented physical experiments and numerical simulations on serrated anchor channels for filling this information gap. To this end, test series on serrated anchor channels were scheduled for determining the structural behavior under longitudinal shear loading $V_{u a, x}$ and to determine how this is influenced by the edge distance [4,5]. Four anchor channels were installed in a concrete slab of height $h$ and positioned orthogonal to the edge, at a prescribed edge distance $c_{a 1,1}$. Each anchor channel was equipped with two riveted anchors installed in a distance $s_{c h b}$. Loading was applied over serrated T-bolts. The anchor channels were loaded until failure. Special attention was paid to the crack formation during loading. Finite element (FEM) simulations, similar to those presented in [6], were performed to study the resulting failure mechanisms. The complex mesh geometry, and the modeling of the physical behavior of the concrete/structural steel as a composite structure in a realistic manner, posed the major challenges here. The interaction between the ductile structural steel and the brittle concrete requires a refined simulation approach. In this study, an experiment with one anchor channel type is used to illustrate the adopted procedure.

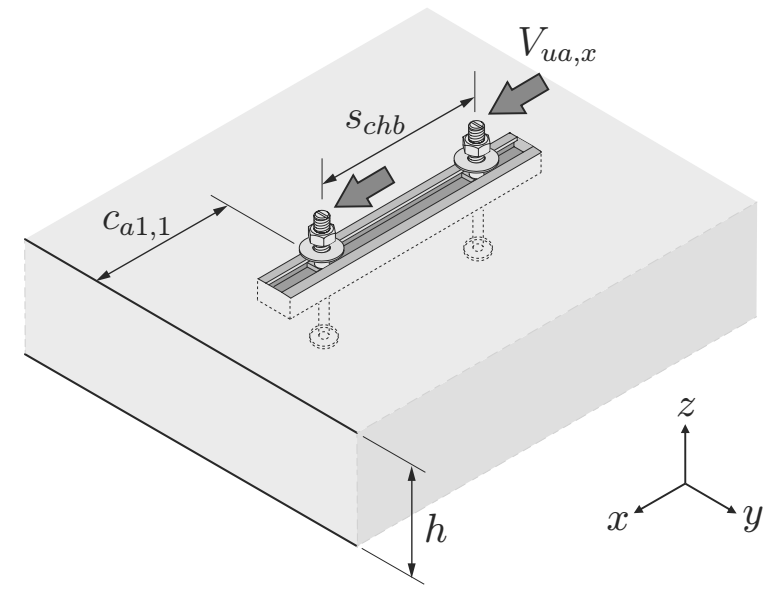

Figure 3: Schematic representation of anchor channel cast-in concrete with two installed T-bolts exposed to shear loading $V_{u a, x}$ in longitudinal channel axis ( $x$-direction).

\section{CALIBRATION OF THE COMPOSITE MODEL}

The numerical composite model consists of the anchor channel comprising structural steel and a concrete slab fabricated from C20/25 concrete. Further, the anchor channel material can be decomposed into the channel material (S275JR) and anchor material (Grade 19MnB4/23MnB4). The T-bolt and washer are made of hardened steel grade 8.8. The whole numerical composite model consists of five parts and four material classes. In addition, all parts are connected by assuming individual contact laws governed by contrasts in material and surface roughness. To approximate the failure mode and ultimate load numerically by means of an FEM approach, each part needs to be calibrated with physical experiments. The calibration experiments were carried out for all parts except the washer, which is assumed linear-elastic since its failure should be avoided. The finite element simulations are performed on the Brutus high-performance computing cluster of ETH Zurich using ABAQUS/Standard and the implemented Newton-Raphson-Solver.

\subsection{Structural steel}

All calibration tests on plain structural steel were carried out at the Quality Control Department of Halfen GmbH in Langenfeld, Germany.

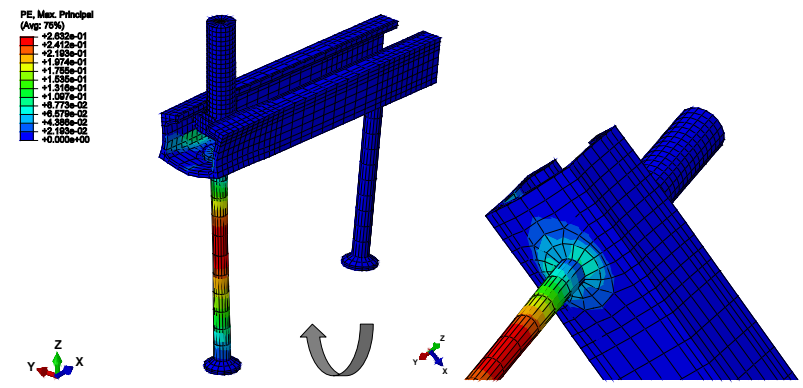

Figure 4: Anchor channel under tensile loading $N_{u a, z}$ with anchor failure (necking and drawing of anchor). Visualization of all plastic strain components (PE, Max. Principal [-]). 


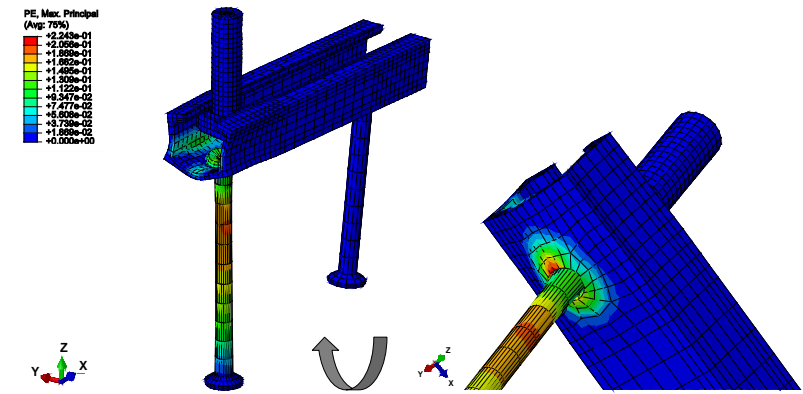

Figure 5: Anchor channel under tensile loading $N_{u a, z}$ with local failure of connection between anchor and channel (buckling and plastification of channel back). Visualization of all plastic strain components (PE, Max. Principal [-]).

The tested channel's dimensions are $b_{c h} \times h_{c h} \times l=52.5 \times 34 \times 250 \mathrm{~mm}$ with a channel thickness $t=4 \mathrm{~mm}$ and height of channel lips $h_{t}=6 \mathrm{~mm}$. The diameter of the round anchor was $12 \mathrm{~mm}$. The installation height was $h_{\text {nom }}=170 \mathrm{~mm}$ with anchor spacing $s=200 \mathrm{~mm}$. The bottom of the anchor is pinned-supported while the top of the T-bolt is fixed in the loading aperture. The anchor channel was tested in tension in the $z$-direction. The load is applied via displacement-control until failure and the resulting load-displacement curves are recorded. Implemented was the "engineering" stress-strain relationship for structural steel calculations.

During these calibration tests, failure of the anchor was manifested in $50 \%$ of the cases (with average ultimate load $N_{u a, z}=62.3 \mathrm{kN}$ ), whereas in the other $50 \%$ failure occurred in the connection between anchor and channel $\left(N_{u a, z}=62.0 \mathrm{kN}\right)$. The contact property between the channel back and the head of the round anchor was simulated by numerous numerical setups, rendering identical failure modes at similar failure loads. The structural steel was modeled plastic assuming a nonlinear material behavior. In Figures 4 and 5 all plastic strain components (PE, Max. Principal) at failure load $F_{u}=N_{u a, z}$ are displayed. The maximum strain components occur at middle height of the anchor, as displayed in Figure 4 , and around the punched hole in the chan- nel back displayed in Figure 5 , The comparison of physical and numerically calculated loaddisplacement curves is illustrated in Figure 10 (left).

\subsection{Concrete}

\subsubsection{Uniaxial tension and compression stress behavior}

The stress-strain curve of concrete under tensile loading [8] may be approximated by a linear-elastic part followed by an exponential function, defined as

$$
\sigma_{t}=f_{t} e^{-\varepsilon_{t} / \varepsilon_{u t}},
$$

where $f_{t}$ is the tensile strength, $\varepsilon_{t}$ is the strain in tension and $\varepsilon_{u t}=G_{f} /\left(L_{s} f_{t}\right)$ a constant depending on $f_{t}$, the fracture energy $G_{f}$ and specific length $L_{s}$. The fracture energy is defined as $G_{f}=G_{f 0}\left(f_{c m} / f_{c m 0}\right)^{0.7}$, where $G_{f 0}$ is a basic value of fracture energy influenced by the maximum grain size $D_{\text {max }} ; f_{c m} \cong 0.85 f_{c}$ is the mean stress value and $f_{c m 0}$ a basic value of cylinder compressive strength.
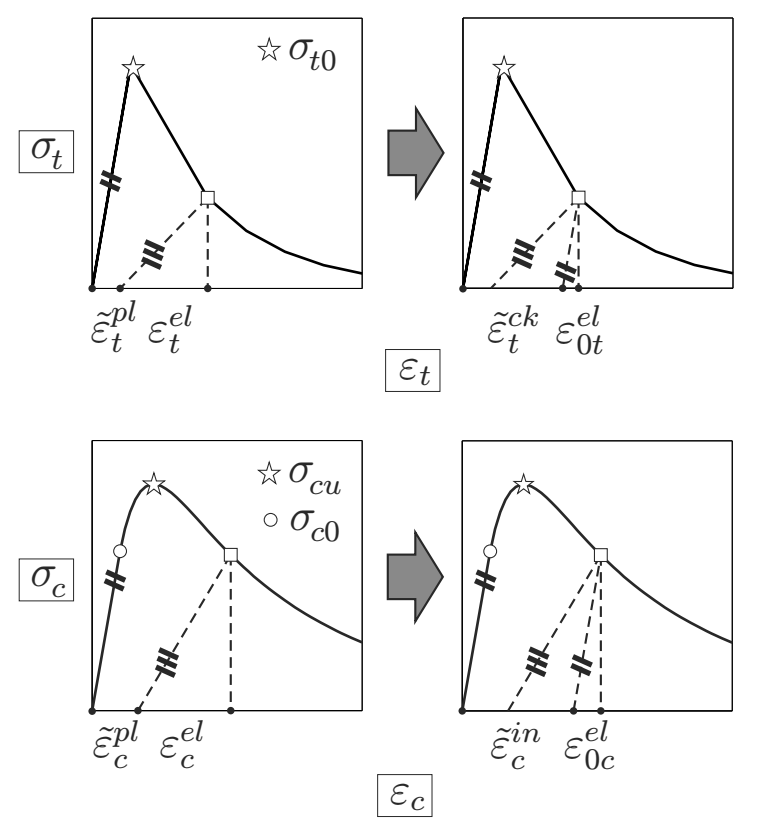

Figure 6: Concrete Damage Plasticity model implemented in ABAQUS according to [9-11].

For approximations of the stress-strain curve of concrete under compression and tensile loading the analytical approach by Sargin [7] and 
Feenstra and DeBorst [8] are adopted. The parabolic compression curve [7] is defined as

$$
\sigma_{c}=\frac{k \eta+(D-1) \eta^{2}}{1+(k-2) \eta+D \eta^{2}} f_{c}
$$

where $D$ is the aspect ratio $\eta=\varepsilon_{c} / \varepsilon_{c 1}, f_{c}$ the compressive strength and $k=E_{0}\left|\varepsilon_{c} / f_{c}\right|>4 / 3$. The strain $\varepsilon_{c 1}$ defines the maximum of the parabolic compression curve. The tensile and compressive stress behavior is modeled using Eqs. (1) and (2), and the input parameter listed in Table 1.

Table 1: Input parameter for numerical modeling of tension and compression stress behavior $\left({ }^{*} D_{\max }=16 \mathrm{~mm}\right.$ is assumed $)$.

\begin{tabular}{cccccc}
\hline $\begin{array}{c}f_{c m 0} \\
{[\mathrm{MPa}]}\end{array}$ & $\begin{array}{c}f_{t} \\
{[\mathrm{MPa}]}\end{array}$ & $\begin{array}{c}f_{c} \\
{[\mathrm{MPa}]}\end{array}$ & $\begin{array}{c}E_{0} \\
{[\mathrm{MPa}]}\end{array}$ & $\begin{array}{c}G_{f 0}{ }^{*} \\
{[\mathrm{~N} / \mathrm{mm}]}\end{array}$ & $\begin{array}{c}\varepsilon_{c 1} \\
{[-]}\end{array}$ \\
\hline 10.0 & 2.9 & 28.1 & $31^{\prime} 400$ & 0.03 & 0.0021 \\
\hline
\end{tabular}

The applied continuum plasticity-based damage model is implemented in ABAQUS, is referred to as the Concrete Damage Plasticity (CDP). The CDP model considers two main failure mechanisms of concrete, tensile cracking and compressive crushing. The yield criterion is fulfilled by including two hardening variables, $\tilde{\varepsilon}_{t}^{p l}$ and $\tilde{\varepsilon}_{c}^{p l}$, referred to as tensile and compressive equivalent plastic strains. These variables activate the failure mechanism caused by tension and compression loading. Under tensile loading the stress-strain response is linearelastic, with a constant initial Young's modulus $E_{0}$, until the failure stress $\sigma_{t 0}$ is reached at onset of micro-cracking (see Figure 6). Beyond the failure stress micro-cracks nucleate, associated with softening of the stress-strain response. Under compression loading the stress-strain response exhibits a similar linear-elastic behavior ( $E_{0}$ const.) until the yielding point, denoted as $\sigma_{c 0}$ (see Figure 6). The parabolic plastic branch of concrete associated with stress hardening is followed by strain softening beyond the ultimate stress $\sigma_{c u}$. Two variables $d_{t}$ and $d_{c} \in[0 ; 1]$ are introduced to incorporate damage into the tensile and compressive behavior, respectively. During deformation the elastic stiffness is degraded by the damage variables such $(1-d) E_{0}$. Note that $d=1.0$ stands for a total loss of strength and $d=0$ designates an undamaged state. The stress-strain relations under uniaxial tension and compression loading can be written as

$$
\sigma_{t}=\left(1-d_{t}\right) E_{0}\left(\varepsilon_{t}-\tilde{\varepsilon}_{t}^{p l}\right)
$$

and

$$
\sigma_{c}=\left(1-d_{c}\right) E_{0}\left(\varepsilon_{c}-\tilde{\varepsilon}_{c}^{p l}\right) .
$$

Conversion of Eqs. (3) and (4) into the effective tensile and compressive stresses leads to

$$
\bar{\sigma}_{t}=\frac{\sigma_{t}}{\left(1-d_{t}\right)}=E_{0}\left(\varepsilon_{t}-\tilde{\varepsilon}_{t}^{p l}\right)
$$

and

$$
\bar{\sigma}_{c}=\frac{\sigma_{c}}{\left(1-d_{c}\right)}=E_{0}\left(\varepsilon_{c}-\tilde{\varepsilon}_{c}^{p l}\right)
$$
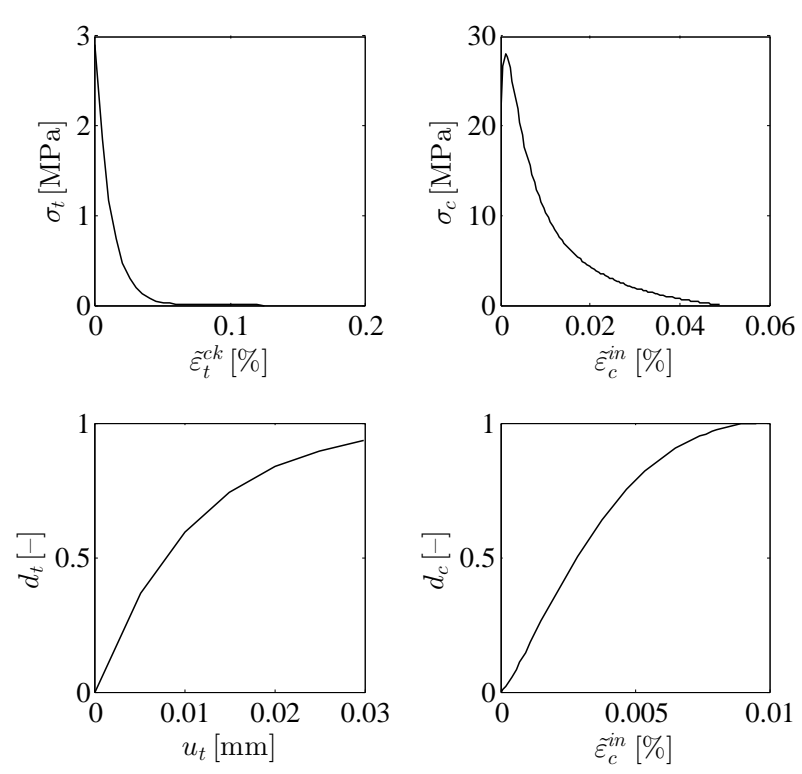

Figure 7: Tensile and compressive concrete behavior implemented in the CDP model used for FEM simulations.

\subsubsection{Tension stiffening: Post-failure stress- strain relation}

The tensile post-failure stress is defined as a function of cracking strain $\tilde{\varepsilon}_{t}^{c k}=\varepsilon_{t}-\varepsilon_{0 t}^{e l}$, 
where $\varepsilon_{t}$ is the strain in tension and $\varepsilon_{0 t}^{e l}=\sigma_{t} / E_{0}$ the elastic strain corresponding to the undamaged material. Plugging these relationships into Eq. (5) leads to the plastic strain in tension

$$
\tilde{\varepsilon}_{t}^{p l}=\tilde{\varepsilon}_{t}^{c k}-\frac{d_{t}}{\left(1-d_{t}\right)} \frac{\sigma_{t}}{E_{0}} .
$$

\subsubsection{Compressive behavior}

Stress hardening is modeled involving the inelastic strain $\tilde{\varepsilon}_{c}^{i n}=\varepsilon_{c}-\varepsilon_{0 c}^{e l}$, where $\varepsilon_{c}$ is the compression strain and $\varepsilon_{0 c}^{e l}=\sigma_{c} / E_{0}$ the elastic strain corresponding to the undamaged material. Plugging these relationships into Eq. (6) leads to the plastic strain under compression

$$
\tilde{\varepsilon}_{c}^{p l}=\tilde{\varepsilon}_{c}^{i n}-\frac{d_{c}}{\left(1-d_{c}\right)} \frac{\sigma_{c}}{E_{0}} .
$$

In absence of tensile and compressive damage, the Eqs. (7) and (8) simplify to $\tilde{\varepsilon}_{t}^{p l}=\tilde{\varepsilon}_{t}^{c k}$ and $\tilde{\varepsilon}_{c}^{p l}=\tilde{\varepsilon}_{c}^{i n}$, respectively.

\subsection{Composite structure}

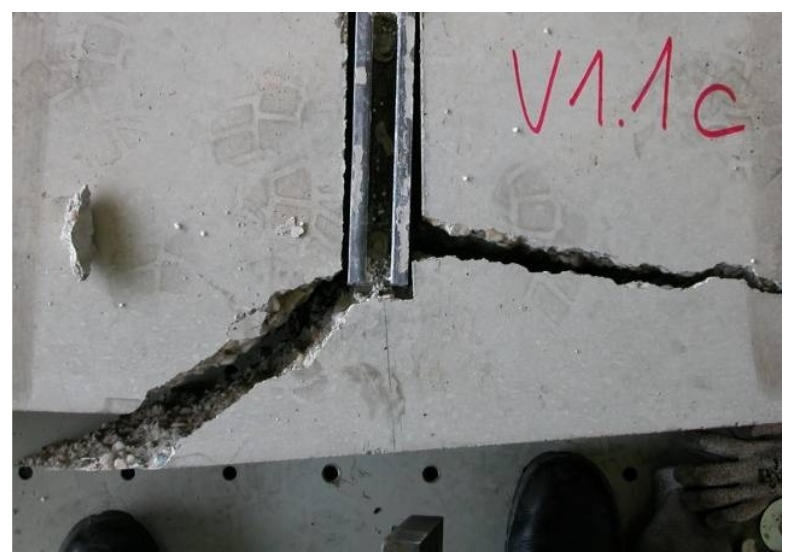

Figure 8: Picture of post-peak cracking pattern after edge failure of the concrete member taken in the physical experiment.

The concrete member of dimensions $x \times y \times z=1280 \times 1280 \times 200 \mathrm{~mm}$ is supported at the bottom $(z=0 \mathrm{~mm})$ on a polished steel surface and at the front $(x=0 \mathrm{~mm})$ at two idealized point supports. The calibration of the composite structure is exercised for one anchor channel. Two T-bolts were installed; one bolt was positioned above the first anchor (close to the edge) and the second one at a distance of $s_{c h b}=100 \mathrm{~mm}$. Both bolts were coupled with a stiff steel plate to distribute the load. The anchor channel was loaded via displacementcontrol in shear in the longitudinal channel axis until failure. The failure mode was identified as a superposition of the dominant concrete edge failure (see Figure 8) and steel failure of the second anchor (not shown).

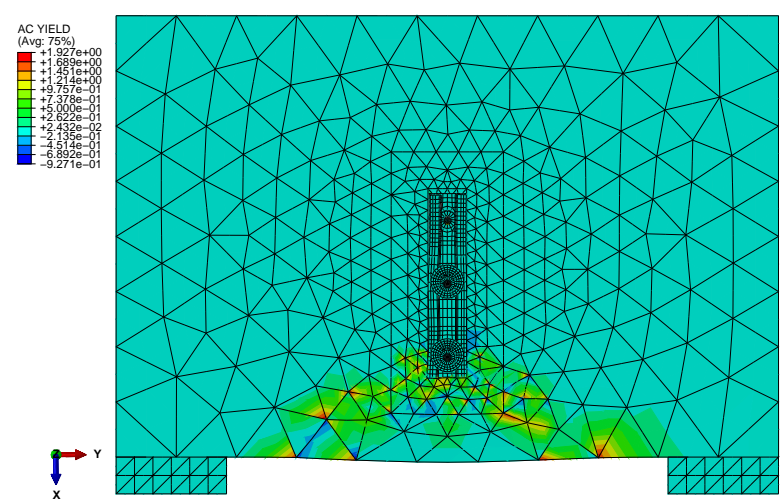

Figure 9: Visualization of cracking pattern of the concrete member at ultimate load (AC YIELD: actual yielding [-]) calculated with FEM (ABAQUS/Standard). No friction is modeled for the bearing support.

In validating the presented numerical composite model the structural steel parts and the concrete slab were assembled into one composite model of size $x \times y \times z=600 \times 900 \times 200 \mathrm{~mm}$. According to CEN/TS 1992-4 [1], the used dimensions allow for formation of a concrete cone in the $y$-direction $\left(y>2 \times 1.5 c_{a 1,1}\right)$.
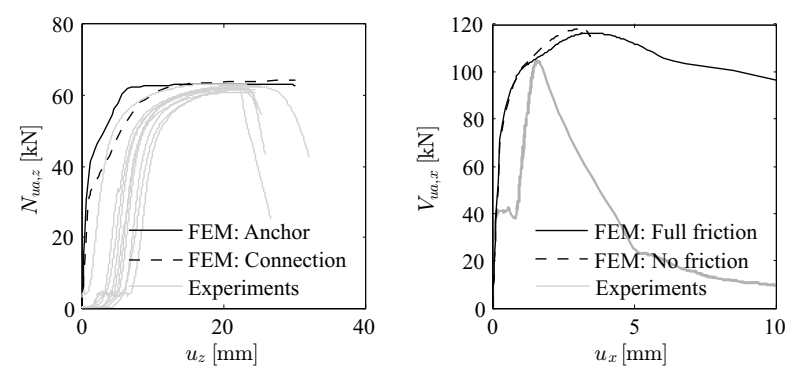

Figure 10: Experimental and numerically calculated load-displacement curves for calibration of plain structural steel (left) and composite structure (right). 
Coulomb friction was assumed between steel and concrete with a friction coefficient $\mu=0.4$. The effect of sliding of the serrated T-bolt in the longitudinal channel axis, associated with plastification of the serrated channel lips, was simplified by a friction coefficient. In addition, two different setups of bearing friction are analyzed, referred to as full friction $(\mu=1.0)$ and no friction $(\mu=0)$. After the numerical simulation the cracking pattern of the concrete member at ultimate load was visualized (see Figure 9). The physical and numerical load-displacement curves were plotted (see Figure 10, right). A good agreement may be observed when comparing both the cracking patterns and the loaddisplacement curves.

\section{LOAD DISTRIBUTION AND CRACK FORMATION UNDER LONGITUDI- NAL SHEAR LOADING}

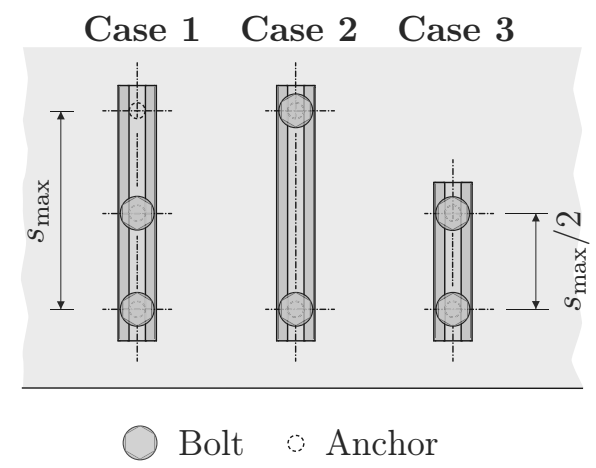

Figure 11: Numerical examples showing three cases of anchor channel installations.

Three numerical examples are designed in delivering some insight as to how the shear load in longitudinal channel axis is distributed after load application on the T-bolt over the anchor channel into the concrete member (see Figure11). The maximum anchor distance was set to $s_{\max }=250 \mathrm{~mm}$ with a channel length of $300 \mathrm{~mm}$. The load was applied over two T-bolts located at varying positions above the anchors. The numerical details and concrete member dimensions were adopted from Section 3.3 . The calculation procedure was split into pre-stressing of the bolts
(ABAQUS/Standard, Newton-Raphson Solver) and shear loading of the composite structure (ABAQUS/Explicit, Dynamic). The Explicit Solver was efficient in calculating the post-peak behavior beyond the point of concrete edge failure. Note that the explicit solution is not based on the solution of static equilibrium with linear system of equations, but rather on the solution of displacements and stresses with time differences of subsequent time steps $(t+\Delta t)$.

The failure mode of interest assumed in this study was the concrete edge failure $V_{u a, x} \equiv V_{R k, c}$ according to [1]. In extracting a rough measure about the load distribution, the shear loads at single anchors were determined according to [1] (see Table 2). Note that $V_{u a, x, i}^{a}$, which is the shear load acting on anchor $i$ of the anchor channel in the longitudinal channel axis, is derived for a group of headed fasteners. The load distributions are computed qualitatively via FEM calculations of the numerical composite model and displayed in Figures 12 and 13 .

Table 2: Summary of shear loads in longitudinal channel axis $V_{u a, x}$ calculated according to CEN/TS 1992-4 [1] for a group of headed fasteners and numerically with FEM for the numerical composite model displayed in brackets (...).

\begin{tabular}{cccc}
\hline & Case 1 & Case 2 & Case 3 \\
\hline$V_{u a, x, 3}^{a}[\mathrm{kN}]$ & 62.9 & 62.9 & - \\
\hline$V_{u a, x, 2}^{a}[\mathrm{kN}]$ & 45.6 & - & 45.6 \\
\hline$V_{u a, x, 1}^{a}[\mathrm{kN}]$ & 27.6 & 27.6 & 27.6 \\
\hline$V_{u a, x}[\mathrm{kN}]$ & 136.0 & 90.5 & 73.1 \\
& $(157.1)$ & $(133.8)$ & $(136.9)$ \\
\hline
\end{tabular}

\section{DISCUSSION}

The shear load $V_{u a, x, i}^{a}$ is partly transmitted over the channel and anchors into the concrete. As can be observed in Figure 12 the cracking patterns of all three cases appear similar. A fracture cone is formed starting from the channel face pushing out off the concrete specimen. The angle of crack formation is similar. Some minor cracks can be observed at the concrete surface around the anchors in the rear face of 
the channel. The dominant deformations occur due to the movement of the fracture cone. No noticeable deformation can be identified behind the middle and rear anchors. Of particular interest are the stress concentrations in the structural steel substructure, namely the channel profile and the anchors. In Case 1 and 2, the major loading part is transmitted into concrete over the rear anchor. In Case 3, both anchors exhibit equal stress concentrations. The illus- trated results are not in agreement with what is suggested in recent design guides. According to CEN/TS 1992-4 [1] for headed fasteners the anchor in the rear transmits the major part of the load (see also Table 2). In AC232 [3], the first three anchors closest to the edge transmit the load equally. For Case 1 each anchor $i$ would transmit $V_{u a, x, i}^{a}=1 / 3 \times V_{u a, x}$, (compare with Table 2).
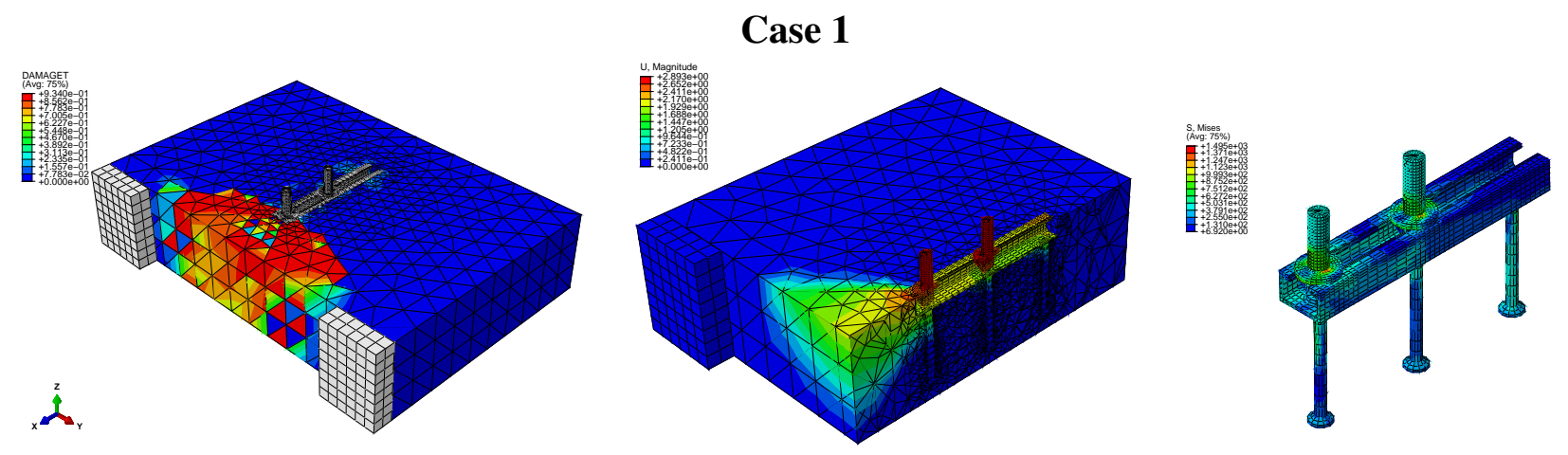

Case 2
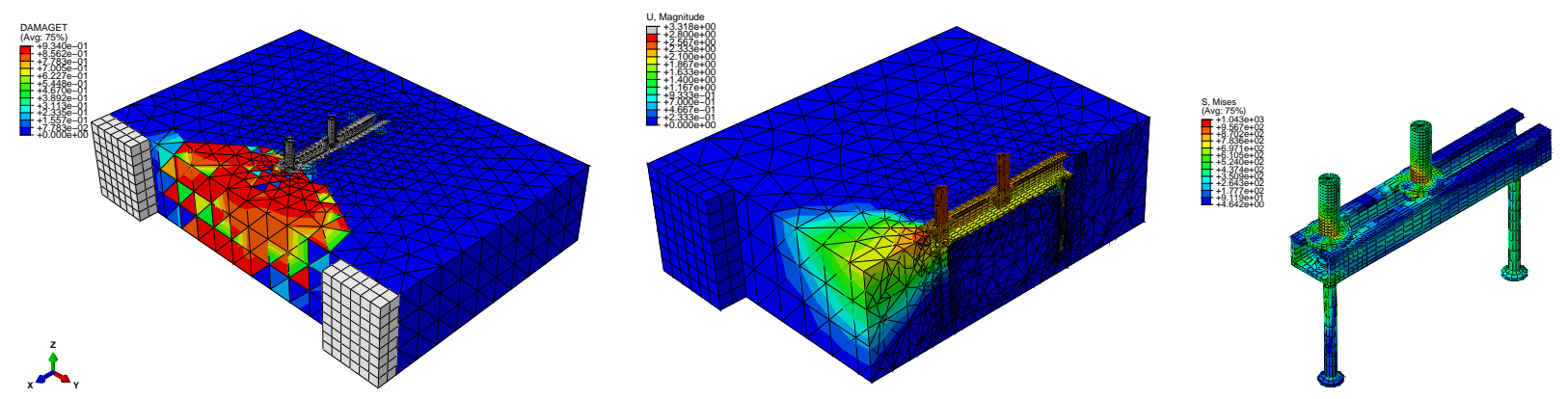

\section{Case 3}
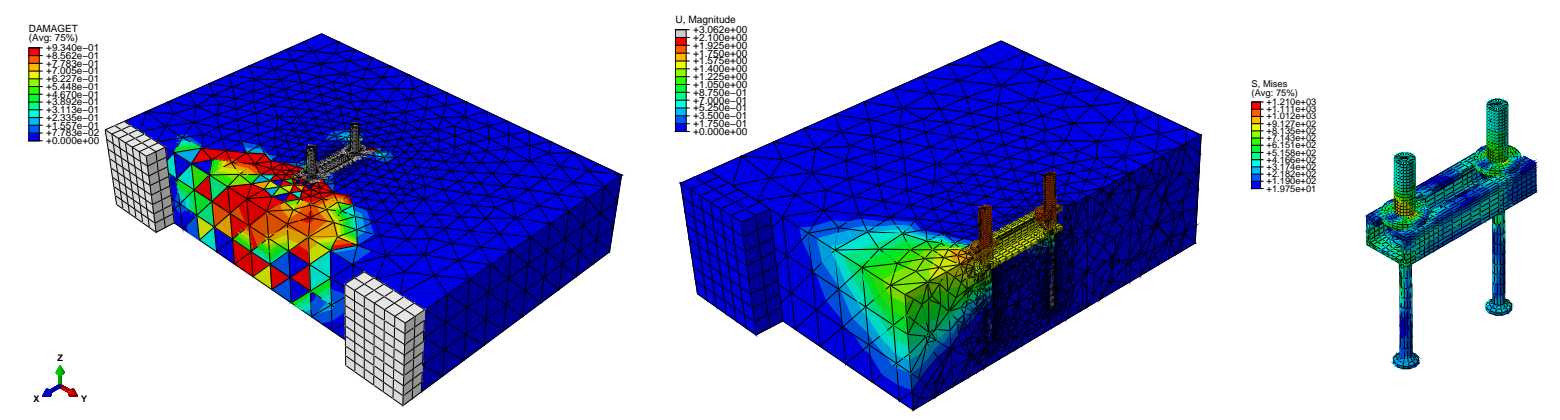

Figure 12: Visualization of cracking patterns (DAMAGET: $d_{t}[-]$, left), magnitude of deformations ( $\mathrm{U}: u=\left(u_{x}^{2}+u_{y}^{2}+u_{z}^{2}\right)^{1 / 2}[\mathrm{~mm}]$, middle) of the composite structure and von Mises stresses (S: $\sigma_{v}=\left(3 / 2 s_{i j} s_{i j}\right)^{1 / 2}[\mathrm{MPa}]$ see also [12], right) of the structural steel substructure, displayed at load level of concrete edge failure $V_{R k, c}$. 

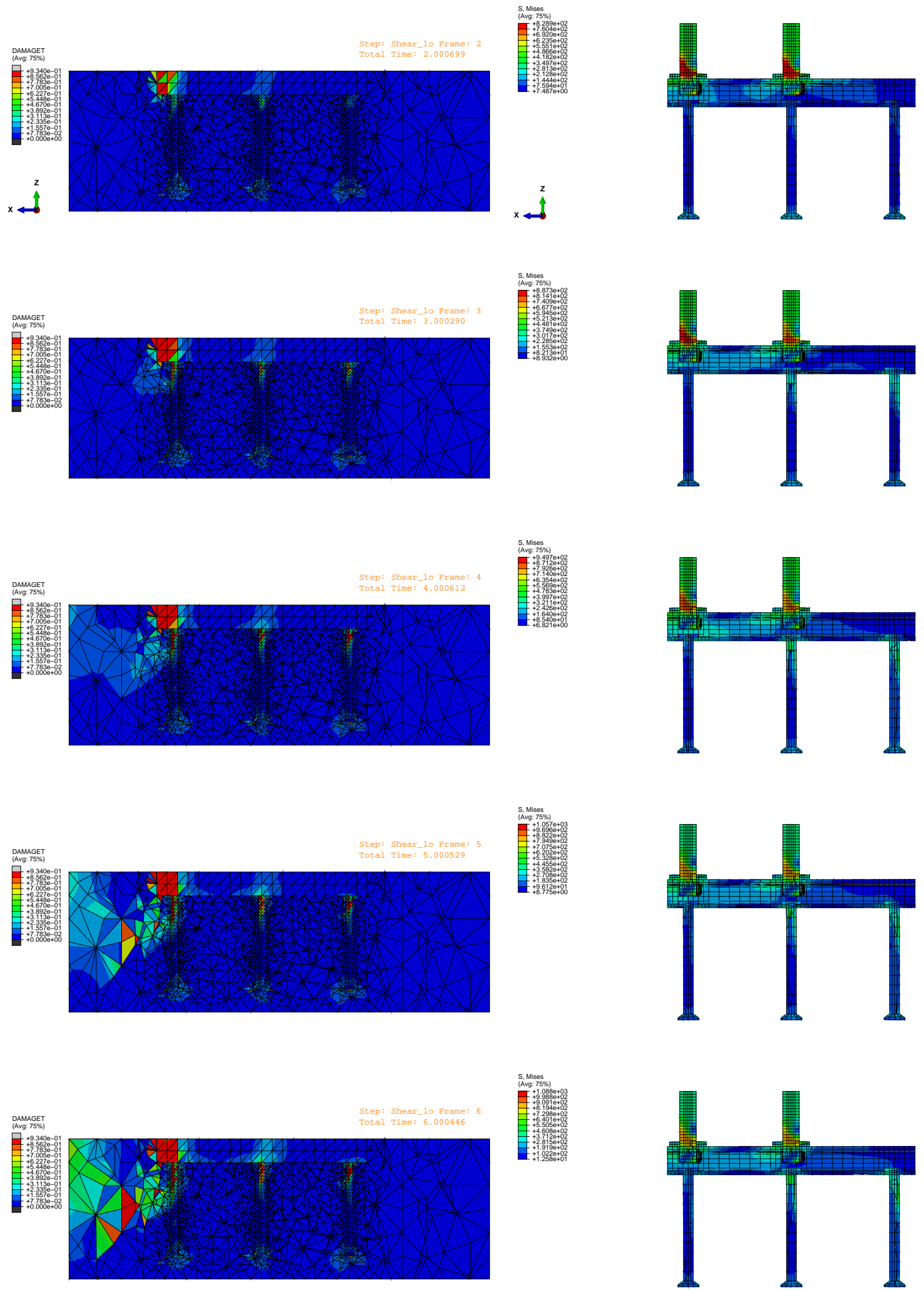

Figure 13: Visualization of cracking formation (Case 1) at the front face of the channel profile in the midplane (DAMAGET: $d_{t}[-]$, left) and the associated von Mises stresses (S: $\sigma_{v}[\mathrm{MPa}]$ see also [12], right) of the structural steel substructure. 
The approach in CEN/TS 1992-4 [1] favors the Cases 1 and 2 and AC232 [3] the Case 3. As can be observed in Figure 13 (left), once the first crack forms due to the longitudinal movement of the anchor channel, the load is transmitted into the rear anchor which shows the stress concentration (see Figure 13 , right). The issue addressed in those numerical examples demonstrates clearly the need for a consistent design method and encourages further detailed investigations in cast-in anchor channels loaded in shear in the longitudinal channel axis.

This work presents the first numerical multiscale investigation on the structural behavior of concrete members due to cast-in anchor channels subjected to sh ear loading in longitudinal channel axis. Apart from anchor channels positioned orthogonally to the edge, further concrete specimen with anchor channels positioned parallel to the edge and in a short distance to the corners need to be addressed in future work.

Calibration of the numerical composite model was successful when applying the DCP model on the concrete member associated with a non-linear behavior of the structural steel anchor channel system and with the contact assumptions that were made. To calibrate the numerical composite model and for deeper investigations on the contact interactions of the different materials and on different modeling scales used (bolt/channel contact: small scale, channel/concrete: large scale) the utilization of the Brutus high-performance computing cluster of ETH Zurich was inevitable.

\section{REFERENCES}

[1] Design of fastenings for use in concrete; German version CEN/TS 1992-4:2009, 28 pp., 2009.

[2] Design of anchorages in concrete. fib Bulletin No. 58; 280 pp., 2011.

[3] Acceptance Criteria for Anchor Channels in Concrete Elements AC232; ICCEvaluation Service, 91 pp., October 2015.
[4] Roik, M. 2010. Supporting the codes: Experimental results confirm theoretical approaches, shown by example of a new generation of anchor channels. 3rd fib International Congress, 9 pp.

[5] Roik, M. 2011. Bearing behaviour of anchorages under shear load close to the edge, here: Anchor channels. fib Symposium PRAGUE, $11 \mathrm{pp}$.

[6] Grosser, P., Eligehausen, R., Ožbolt, J. 2010. 3D FE analysis of anchor channels and headed stud anchors under shear load close to the edge. In B. H. Oh, et al. (Eds) Fracture Mechanics of Concrete and Concrete Structures Recent Advances in Fracture Mechanics of Concrete; pp. 560-567.

[7] Sargin, M. 1971. Stress-strain relationships for concrete and the analysis of structural concrete section, Solid Mechanics Division, University of Waterloo.

[8] Feenstra, P. H. and De Borst, R. 1961. A composite plasticity model for concrete. International Journal of Solids and Structures 33(5):707-730.

[9] Hillerborg, A., Modeer, M. and Petersson, P. E. 1976. Analysis of Crack Formation and Crack Growth in Concrete by Means of Fracture Mechanics and Finite Elements, Cement and Concrete Research, 6: pp. 773-782.

[10] Lubliner, J., Oliver, J., Oller, S., and E. Oñate, 1989. A Plastic-Damage Model for Concrete, International Journal of Solids and Structures, 25: pp. 299-329.

[11] Lee, J., and Fenves, G. L. 1998. PlasticDamage Model for Cyclic Loading of Concrete Structures, Journal of Engineering Mechanics, 124(8): pp. 892-900.

[12] Hill, R. 1998. The Mathematical Theory of Plasticity, Oxford University Press, New York (11th ed., 1998, 357 pp.). 